\title{
Familial histiocytic reticulosis (familial haemophagocytic reticulosis)
}

\author{
VALERIE J. MARRIAN AND N. G. SANERKIN \\ From the Departments of Child Health and Pathology, University of Bristol
}

SYNOPSIS The clinical and pathological findings are recorded in two siblings who died in early infancy from familial histiocytic ('haemophagocytic') reticulosis. The nature of this condition is obscure but probably represents a primary histiocytosis. The only other family on record is that described by Farquhar and Claireaux (1952) and by Farquhar, Macgregor, and Richmond (1958).

This unusual disorder was first described by Farquhar and Claireaux (1952), who recorded a family with three siblings the first two of whom died in early infancy, the third sibling recovering from what appeared to be a mild form of the disease. Subsequently, Farquhar et al. (1958) reported a fourth sibling in the same family who died from an identical condition. In all three fatal cases, considered by these authors to represent the homozygous state in a familial disorder, the infants had developed anorexia, vomiting, irritability, and pallor during the first three months of life and were found to have hepato-splenomegaly with progressive anaemia, granulocytopenia, and thrombocytopenia. In all cases, the peripheral blood showed numbers of smudged cells and a small proportion of atypical mononuclear cells. The surviving sibling had developed severe anorexia at the age of 10 weeks. Smudged cells and atypical mononuclears were found in her blood films until the age of 18 months, but she never had any hepato-splenomegaly and had since remained quite healthy, apparently representing the heterozygous state of this disorder.

Necropsy in the three fatal cases was reported to have shown a remarkable proliferation of histiocytes throughout the reticulo-endothelial system, with prominent phagocytosis of blood cells, especially of the red cells. The authors of the original paper concluded that the condition was not Letterer-Siwe's 'non-lipid reticulo-endotheliosis'. Their suggestion that it might be identical with histiocytic medullary reticulosis was refuted by Marshall (1956).

The present paper describes a second family in which the two offspring died in early infancy from a condition identical with that described by Farquhar et al. $(1952,1958)$. Although the first case was correctly diagnosed as a malignant reticulosis, the exact

Received for publication 7 August 1962. nature of the disease was not firmly established until after the death of the second sibling, when a search of the literature was made with special reference to histiocytic medullary reticulosis and Letterer-Siwe disease. Because of this, the detailed haematological investigations performed by Farquhar et al. (1958) could not be carried out on these infants, in particular the red-cell survival times and the detection of autoantibodies. Unfortunately, the parents themselves on religious grounds declined to undergo any tests for determination of their own red-cell survival times.

The father, aged 22 at the birth of the first child, had been diagnosed as a case of pulmonary tuberculosis two and a half years previously. He was treated with I.N.A.H. and P.A.S. and is now apparently healthy. The mother, aged 20 , is an epileptic controlled by treatment with phenobarbitone and for a period with diamox and paradione. They are not blood relatives. On neither side of the family has there been similar illness or death in infancy.

The parents consented to simple tests on their blood, the results of which are given below:

Father Hb $99 \%$, R.B.C. 4,600,000/c.mm., P.C.V. $42 \%$. W.B.C. 10,800/c.mm. (polymorphs 5,000/c.mm.). Direct Coombs test negative.

Mother Hb $82 \%$, R.B.C. 4,200,000/c.mm., P.C.V. $35 \%$, W.B.C. 9,200/c.mm. (polymorphs 6,200/c.mm.). Direct Coombs test negative.

CASE 1 The first-born, a male infant, was delivered spontaneously at term after a normal pregnancy (birth weight $7 \mathrm{lb}$. $11 \mathrm{oz}$.). He was given B.C.G. at the age of 6 days. He appeared quite healthy until the eighth week when he was admitted to hospital with one day's history of irritability, photophobia, anorexia, projectile vomiting, and grunting respiration. He was pale, shocked, and miserable (temperature $102 \cdot 7^{\circ} \mathrm{F}$., pulse $160 / \mathrm{min}$., respiration $48 / \mathrm{min}$.). He had mild coryza but no evidence of any other infection. There was no cutaneous rash, and no lymphadenopathy or hepato-splenomegaly was noted initially. Lumbar puncture showed normal cerebrospinal 
fluid. Haemoglobin was $58 \%$; a blood film was orthochromic with some anisocytosis; platelets appeared to be present in normal numbers; reticulocytes were less than $1 \%$; W.B.C. $4,400 /$ c.mm. Plasma was not icteric. The blood group was $\mathrm{O}$ Rh (D) positive. The direct Coombs test was negative, as was the Paul Bunnell test. A tibial marrow puncture showed normoblastic erythropoiesis, with a myeloid-erythroid ratio of $4: 3$. A non-catheter specimen of urine contained albumin + , scanty white cells, and culture yielded Bact. coli and enterococci. Staph. aureus, resistant to penicillin only, was isolated from the nose. He was treated empirically with tetracycline, $62.5 \mathrm{mg}$. six-hourly, and transfused with $250 \mathrm{ml}$. of whole blood after which the haemoglobin rose to $94 \%$. An intravenous pyelogram, performed on the fourth day after admission, showed no significant abnormalities, but a soft tissue mass was noted in the left upper abdomen. By the tenth day the liver was enlarged two fingerbreadths and a large mobile swelling was felt in the left hypochondrium which was presumed to be the spleen. On the 17 th day the haemoglobin had fallen to $54 \%$ and the red cells showed hypochromia and anisocytosis; W.B.C. $6,200 /$ c.mm. (polymorphs $750 /$ c.mm.) and 160 smudged cells per 100 white cells. A purpuric rash was noted about this time. An exploratory laparotomy performed on the 20th day showed considerable ascites and a very large soft spleen which was removed. His postoperative condition was poor. He collapsed a few hours later and died the same day.

The excised spleen weighed $157 \mathrm{~g}$. and was large and soft, with a smooth capsular surface free from adhesions. The cut surface was homogeneous, soft and pink, with indistinct Malpighian bodies. Histological examination showed changes identical with those found in the spleen of the second case, and will be described later.

Post-mortem examination The body was that of a very pale male infant weighing $5,365 \mathrm{~g}$. There was no jaundice, oedema, or external haemorrhage. The abdomen was protuberant, with a sutured surgical wound on the left side. The abdominal cavity contained about $10 \mathrm{ml}$. of blood-stained fluid with a few clots. The pleural and pericardial cavities contained small amounts of clear yellow fluid.

The significant naked-eye abnormalities in the internal organs were as follows: A haematoma of about $10 \mathrm{ml}$. was found in the splenic bed. The liver was enlarged and weighed 379 g.; it was pale brown in colour, firm and fibrous in texture, but smooth in outline. The lymph nodes of the neck and abdomen were slightly enlarged, soft, discrete, and pink. The bone marrow in the sternum and femur appeared normal. The other organs were normal though pale. A Meckel's diverticulum was present.

The histological findings were similar to those of Case 2 and will be described later.

CASE 2 The second child, a girl, was born one year later after an uneventful pregnancy and delivery (birth weight $8 \mathrm{lb} .6 \mathrm{oz}$.). B.C.G. was given at the age of 7 days. At the age of 4 months she became unusually quiet and sleepy. She developed a febrile illness associated with vomiting and nasal 'snuffles' but no other respiratory symptoms. One week later her eyes became puffy, and within a few days the swelling had involved the entire face and neck. She was admitted to hospital after two weeks illness looking very ill (temperature $104.4 \mathrm{~F}$., pulse $\overrightarrow{\vec{F}}$ $184 / \mathrm{min}$., respiration $62 / \mathrm{min}$.). She was extremely pale with a bluish tinge of the lips. Her face was grossly oedematous and there was a sero-sanguinous nasal dis- $\frac{\bar{\sigma}}{\bar{C}}$ charge. The abdomen was very distended with demon- $\frac{D}{T}$ strable ascites. The spleen was palpable three $\unrhd$ fingerbreadths and the liver two fingerbreadths below the costal margin. Haemoglobin was $39 \%$, W.B.C. 2,700/c.mm. (polymorphs $720 / \mathrm{c}$.mm., reticulocytes $2 \cdot 2 \%$ ). platelets $6,000 / \mathrm{c} . \mathrm{mm}$. The blood film showed mild $\vec{\overrightarrow{ }}$ generalized hypochromia with normocytosis but occa- $\vec{\omega}$ sional macrocytes, scanty platelets, and granulocytopenia with an increase in juvenile forms. There were many? smudged cells and some atypical mononuclear cells with $\vec{\sigma}$ heavily stained distorted nuclei. The blood group was A Rh (D) positive. Coombs test was not performed. Total of serum proteins were $3 \mathrm{~g} .100 \mathrm{ml}$. with low albumin and $\mathrm{O}$ globulin. The serum cholesterol level was $260 \mathrm{mg} . / 100 \mathrm{ml}$., and the total serum lipids $2.34 \mathrm{~g}$. $/ 100 \mathrm{ml}$. The urine $\vec{c}$ showed a trace of albumin, normal sediment, and no ฏ cytomegalic inclusion bodies were found. A nasal swab yielded coagulase-negative Staph. aureus resistant to penicillin only. She was treated empirically with penicillin $\rightarrow$ and streptomycin and transfused with $190 \mathrm{ml}$. of whole blood. She deteriorated progressively with falling haemo- $\omega$ globin, increasing splenomegaly and abdominal disten- $\square$ sion. She developed ecchymoses in the eyelids and bled from the ears. A fine purpuric rash appeared over the abdomen and thighs. On the fourth day after admission the polymorphonuclear count had fallen to less than $\frac{0}{\Phi}$ $50 / \mathrm{c} . \mathrm{mm}$. and platelets were not detectable. A tibial $\mathrm{Q}$ marrow puncture showed moderate cellularity with very $\overrightarrow{\overrightarrow{0}}$ active erythropoiesis, predominantly normoblastic but $\frac{0}{3}$ with minor megaloblastic changes. The granulocytic $\vec{P}$ series was depressed, showing mainly promyelocytes, reduced numbers of myelocytes, very few juvenile polymorphs and extremely few segmented cells. Megakaryo- $\frac{\overline{0}}{0}$ cytes were scanty with abnormal nuclei and practically no $\overline{0}$ platelets. Reticulum cells were slightly increased in 3 . numbers and many showed pale vacuolated cytoplasm. No malignant cells were seen and there was no evidence 3 of leukaemia. She died on the eighth day after admission at the age of 5 months.

Post-mortem examination The body was that of a $\frac{D}{2}$ female infant weighing $6,500 \mathrm{~g}$., with marked pallor, ecchymoses over the eyelids and groins, and a generalized $\mathrm{N}$ purpuric rash maximal over the trunk and thighs. There was no obvious jaundice. Generalized pitting oedema was $\mathbb{O}$ present. The abdomen was prominently distended. Small N collections of straw-coloured fluid were found in all $\sigma$ the serous cavities, with subserous petechial haemorrhages.

The significant naked-eye abnormalities in the internal $\mathbb{D}$ organs were as follows. The spleen was grossly enlarged, weighing $195 \mathrm{~g}$. and extending down to the left iliac fossa. It was firm, with a smooth capsular surface without any pericapsular adhesions and a uniform purplish-red cut $\stackrel{\mathcal{P}}{\mathscr{Q}}$ surface with indistinct Malpighian bodies. The liver was $\mathbb{\mathbb { Q }}$ moderately enlarged and weighed 440 g., with sharp $\bar{\sigma}$ margins and smooth capsular surface; the cut surface 
showed no abnormality apart from obvious pallor. The lymph nodes were not enlarged to any remarkable degree; they were discrete, soft and rather oedematous, with a pinkish cut surface. The bone marrow was uniformly deep red throughout. An incidental finding was the presence of bilateral hydroureter and hydronephrosis due to congenital uretero-vesical stenosis, without evidence of any pyelonephritis.

\section{HISTOLOGY}

The microscopical lesions were identical in the two infants and represented a diffuse histiocytic proliferation throughout the reticulo-endothelial system, in the spleen, bone marrow, liver, and lymphoid tissues. A variable degree of histiocytic infiltration was found in all other tissues and organs, where it showed a paravascular distribution. In the lymph nodes the normal structural framework remained intact, although the tissues were overgrown by collections of histiocytic cells most prominent in and around the sinuses (Fig. 1). In the liver there was marked histiocytic proliferation in the portal tracts, with lesser infiltration in the sinusoids (Fig. 2). In the spleen the pulp was markedly expanded due to similar histiocytic proliferation. The bone marrow showed extensive diffuse infiltration by histiocytes, with considerable displacement of all normal haemopoietic elements, particularly in case 2 . Residual haemopoietic islands showed predominance of normoblasts, with scanty myeloid cells and rare megakaryocytes. Sections from the brain, suprarenals, pancreas, heart, salivary glands, lungs, kidneys, and gonads showed varying degrees of infiltration by histiocytes, mainly paravascular in distribution and, with the exception of the lung (Fig. 3), nowhere as prominent as in the liver, spleen, lymph nodes, or bone marrow. Neither in the bone marrow nor at any other site was there any appreciable increase in fibrous tissue, although a slight increase in reticulin network was noted.

The proliferating histiocytes (Fig. 4) measured about 15 to $35 \mu$ in diameter and showed marked phagocytic activity, ingesting all sorts of matter, including erythrocytes, nucleated cells of various types, naked nuclei and nuclear debris, as well as non-specific lipid subśtances. Among the ingested matter, the erythrocytes were by far the most numerous. Many of the histiocytes contained sudanophilic material (Fig. 5) histochemically shown not to be cholesterol, cerebroside, or phosphatide. Many of the cells also contained P.A.S.-positive material in their cytoplasm. Haemosiderin deposits were scanty and slight, being confined to the cytoplasm of an occasional histiocyte with no free iron pigment in the tissues. No acid-fast bacilli were seen in ZiehlNeelsen preparations. The majority of the histio-

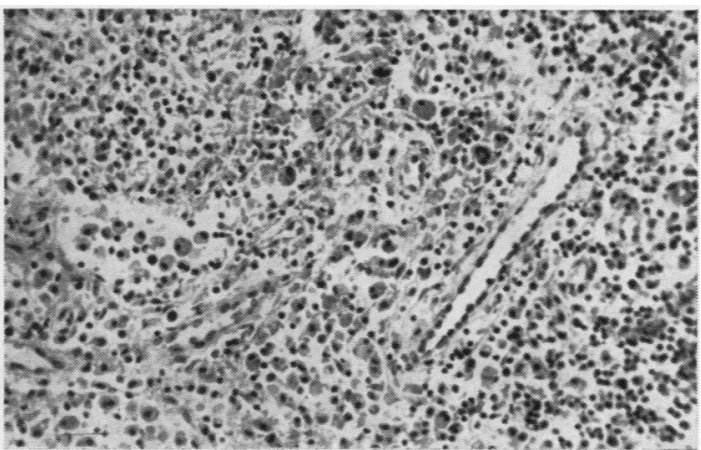

FIG. 1. Diffuse infiltration of lymph node by histiocytes. The cytoplasm is abundant and frequently contains ingested erythrocytes and nucleated cells; nuclei are pyknotic and eccentric. Haematoxylin and eosin $\times 160$.

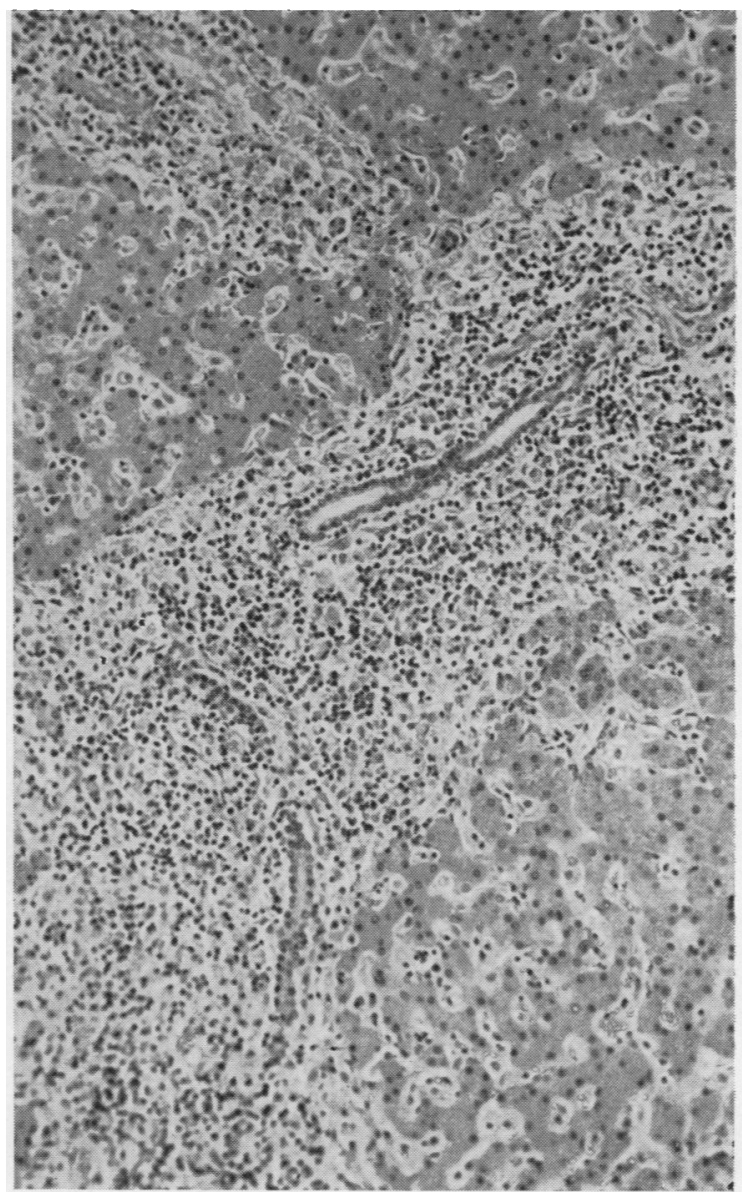

FIG. 2. Histiocytic infiltration of portal tracts in liver; occasional histiocytes are also present in hepatic sinusoids. Haematoxylin and eosin $\times 95$. 


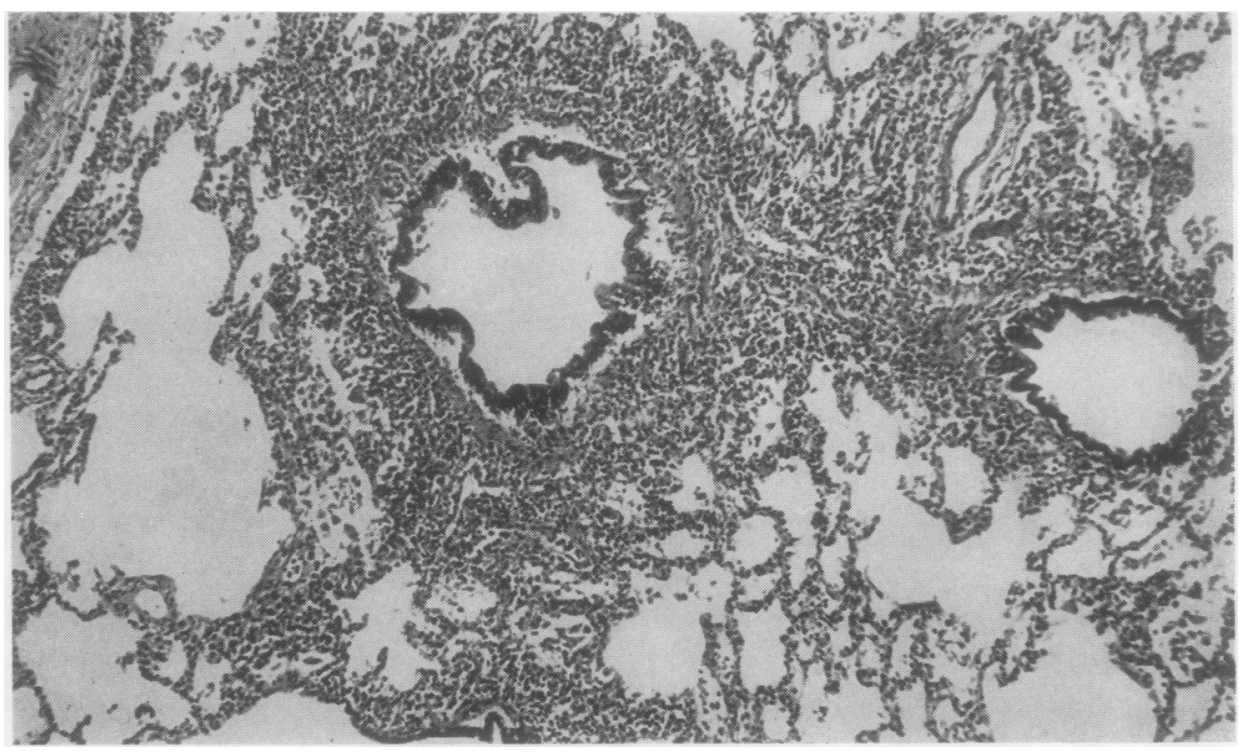

FIG. 3. Peribronchial and perivascular infiltration by histiocytes in lung. The infiltrate is typically interstitial, not bronchopneumonic. Haematoxylin and eosin $\times 95$.

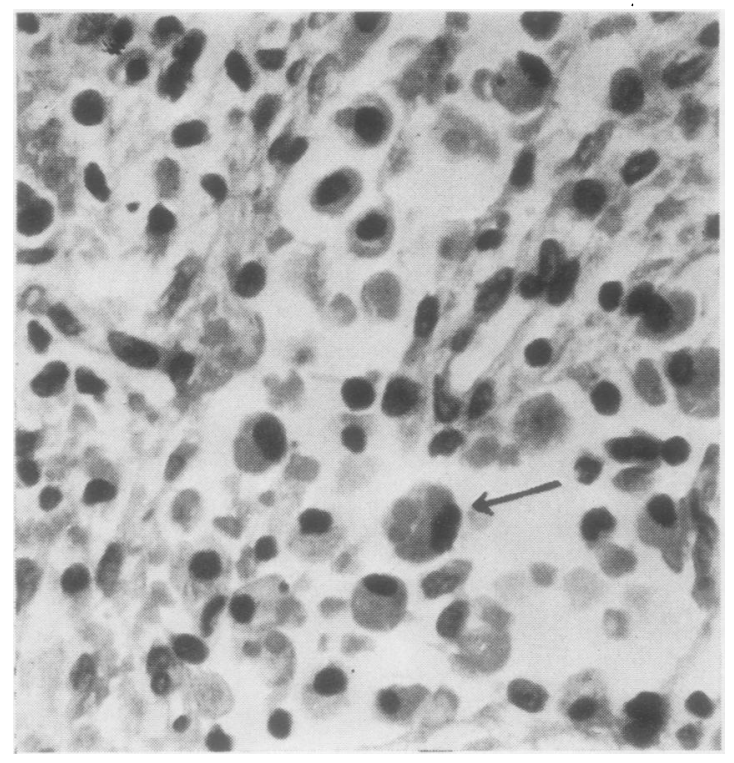

FIG. 4. High-power view of histiocytes in the medulla of a lymph node. Arrow points at a histiocyte containing several erythrocytes. Haematoxylin and eosin $\times 600$.

FIG. 5. Cuff of sudanophilic histiocytes around a cerebra arteriole. Frozen section of brain stained Sudan III. $\times 15$. 
cytes were fully differentiated and mature, with scanty primitive cells and few mitoses.

A differential lipid analysis was performed on the spleen of case 2 (Table I) and the results were not considered to be abnormal. The slightly raised sphingomyelin, $1.3 \%$ dry weight, was not considered to be of any special significance, since in a NiemannPick spleen it was raised to $13 \cdot 2 \%$. The sudanophilic material within the histiocytes would thus appear to originate from phagocytosis of non-specific lipid material from the plasma and partly from the breakdown of ingested cells.

\section{TABLE I}

DIFFERENTIAL LIPID ANALYSIS OF THE SPLEEN $\%$ Dry Basis

Normal Spleen Spleen from Case 2

Total lipid
Total lipid hexose
Neutral hexose
Lipid hexosamine
Lipid sulphur
Free cholesterol
Ester cholesterol
Total phosphatide
Lecithin
Sphingomyelin
Cephalin A
Cephalin B
Water

The lipid analysis on the spleen is not considered abnormal. Although the neutral hexose and lipid sulphur have not been done on the normal spleen, the results for case 2 are unlikely to be abnormal. The raised sphingomyelin is probably due to the formol. This was as high as 13.2 in a Niemann-Pick spleen.

\section{DISCUSSION}

Familial histiocytic reticulosis must be a rare condition, since there is no record of any other cases apart from the family described by Farquhar et al. $(1952,1958)$ and the family now recorded. The possible mode of inheritance has been discussed by Farquhar et al. (1958) and transmission would appear to be by a recessive gene, manifesting itself in a fatal form when homozygous and remaining asymptomatic when heterozygous. It is possible, however, that isolated cases may well be missed, being labelled as 'obscure malignant reticuloses', as indeed were the first siblings in the two families so far described. Further, some cases may be diagnosed as Letterer-Siwe's reticulo-endotheliosis which is perhaps the condition most likely to be confused with familial histiocytic reticulosis.

In the differential diagnosis of our cases we considered the lipid-storage diseases, acute histiocytic leukaemia, and 'reticulosis'. There was no evidence of a leukaemic process in the blood or bone marrow films during life and, although post-mortem histological sections showed infiltrations similar to those occurring in the leukaemias, the infiltrating cells were almost exclusively mature histiocytes and not primitive forms such as might be expected in an acute leukaemia. A lipidosis was confidently excluded on the necropsy findings, both by the histological picture and by the differential lipid analysis of the spleen. Letterer-Siwe's reticulo-endotheliosis was excluded, because in this condition the proliferations contain primitive reticulum cells as well as histiocytes, and tend to produce focal lesions often involving the skeletal tissues.

Familial histiocytic reticulosis is a condition characterized by diffuse histiocytosis with prominent phagocytosis, especially of blood cells. The aetiology remains obscure. It might be a primary disorder of the reticulo-endothelial system, a primary reticulosis, or it could be reactive, for example, to auto-immunization of blood cells or to some histiocytosis-provoking infection. The evidence concerning a possible autoimmune process has been presented by Farquhar $e t$ al. (1958) but is difficult to assess. The direct Coombs test was not consistently positive, being negative in two of their fatal cases. This test was also negative in our case 1 , the only instance in which it was performed on our own patients. The markedly reduced red-cell survival time noted by Farquhar et al. (1958) in their cases could well indicate destruction of red cells by the phagocytic histiocytes and need not implicate any auto-immunization process. A reactive histiocytosis is known to occur in some infections, for instance, enteric fever. We have no evidence that these infants were suffering from any ascertainable infection. Nevertheless, the bacteriological investigations were far from adequate, and the possibility of some unusual infection should be borne in mind in any future cases.

We consider it probable that the histiocytic proliferation in this condition is indeed primary, the observable haematological complications being merely secondary effects. The anaemia, granulocytopenia, and thrombocytopenia are probably the result of two separate processes, the first undoubtedly being the phagocytosis of formed blood elements by the histiocytes; the second, probably no less important, is replacement of marrow by the extensive histiocytic infiltration of the bone marrow.

We are indebted to Professor A. V. Neale for agreeing to publish these cases, to Dr. N. J. Brown for the morbid anatomical and histopathological details of the first case, to Mr. A. Tingey for the differential lipid analysis of the spleen, and to Mr. D. White for the photography.

\section{REFERENCES}

Farquhar, J. W., and Claireaux, A. E. (1952). Arch. Dis. Childh., 27, 519.

MacGregor, Agnes R., and Richmond, J. (1958). Brit. med. J., 2, 1561 .

Marshall, A. H. E. (1956). J. Path. Bact., 71, 61. 